\title{
Long-lasting cortical plasticity in the object naming system
}

\author{
Miranda van Turennout ${ }^{1,2}$, Timothy Ellmore ${ }^{1}$ and Alex Martin ${ }^{1}$ \\ ${ }^{1}$ Laboratory of Brain and Cognition, National Institute of Mental Health, Building 10, Room 4C104, 10 Center Drive MSC 1366, Bethesda, \\ Maryland 20892-1366, USA \\ 2 Current address: Max Planck Institute for Psycholinguistics, Wundtlaan 1, NL-6525 XD Nijmegen, The Netherlands \\ Correspondence should be addressed to M.V.T. (Miranda.VanTurennout@mpi.nl)
}

\begin{abstract}
A single exposure to an object can produce long-lasting behavioral change. Here, using eventrelated functional magnetic resonance imaging (fM RI), we provide evidence for long-lasting changes in cortical activity associated with perceiving and naming objects. In posterior regions, we observed an immediate (30-second) and long-lasting (3-day) decrease in neural activity after brief (200-ms) exposure to nameable and nonsense objects. In addition, slower-developing decreases in left inferior frontal activity were observed concurrently with increases in left insula activity, only for nameable objects. These time-dependent cortical changes may reflect two distinct learning mechanisms: the formation of sparser, yet more object-form-specific, representations in posterior regions, and experience-induced reorganization of the brain circuitry underlying lexical retrieval in anterior regions.
\end{abstract}

Naming an object once facilitates naming that same object again. Behavioral studies demonstrate that this form of learning, commonly called 'repetition priming', is immediate, and can last for long periods of time ${ }^{1,2}$. Speeded naming responses can be observed in normal individuals up to 48 weeks after a single exposure to an object ${ }^{3}$, suggesting a permanent change. Long-lasting facilitation of object naming is normal in profoundly amnesic patients, suggesting that this form of learning is not dependent on the medial temporal lobe memory system ${ }^{4}$. The neural mechanisms underlying this long-lasting form of learning are largely unknown. Studies of cortical plasticity suggest that cortical representations are continuously modified as a function of experience throughout life ${ }^{5}$. Consistent with this idea, single-cell recording from monkey cortex ${ }^{6,7}$ and functional brain imaging of human adults ${ }^{8,9}$ show decreases in neural activity in occipitotemporal regions of the brain following object repetition. It has been suggested that these reductions in neural activity may reflect the creation of sparser, yet more object-specific representations ${ }^{10,11}$. According to this view, as object-form-specific features are learned through experience, the network of cells that are well tuned to this information will keep responding, whereas cells not specifically tuned will drop out. Hence, the total number of cells responding to a specific stimulus will be smaller, but more selective, and total activity will be decreased as a function of repeated experience with objects. In previous studies, however, the repetition lag has been short (that is, seconds to minutes) and it is an open question whether this neural mechanism mediates the longlasting behavioral learning effect observed in object naming.

In object naming, qualitatively different types of information have to be retrieved from memory ${ }^{12}$. The recognition of the object's form and the activation of its semantic properties trigger the retrieval of lexical information (that is, the object's name). Data from behavioral and electrophysiological studies suggest that these properties are distinct and retrieved at high speed during object naming ${ }^{12,13}$. Repetition priming could, in principle, originate at any one of the different processing levels of the object-naming system, or in their interaction. Indeed, although some investigators have argued that repetition priming in object naming reflects changes in the perceptual representation ${ }^{1}$, others suggest that this form of learning is mediated by changes at the lexical-semantic levels of representation ${ }^{14}$.

Here we investigate, using fMRI, the modulation of neural activity at perceptual and higher-order levels of the object naming system as a function of object repetition after long and short time intervals. We proposed that if repetition priming is associated with the creation of sparser, form-specific object representations, long-lasting reductions in activity should be observed in occipitotemporal regions after experience with nameable objects, as well as with objects that do not have pre-existing representations in memory (nonsense objects). If learning also involves lexical-semantic levels of representation, distinct neural changes should be observed for repeated object naming in higher-order regions of the object-naming pathway.

Eight naive subjects were presented with pictures of nameable and nonsense objects outside the scanner. The subjects' task was to look carefully at every picture and to name the real objects. The pictures were presented briefly and were intermixed to ensure that subjects paid attention to both nameable and nonsense objects. Three days later, fMRI time series were obtained while subjects saw these pictures again (3-day delay), saw pictures not previously presented (novel), and saw a repetition of each novel picture 30 seconds after initial exposure (30-second delay). All pictures were presented rapidly and were randomly intermixed. Because all event-types (novel, 30-second delay, 3-day delay) were included in the same scanning session, fMRI results were not confounded by differences in signal intensity among sessions. Moreover, because we presented the pictures for only $200 \mathrm{~ms}$ and used an intermixed (event-related) design, subjects were prevented from anticipating and changing strategies for processing the different event types. Therefore, differential results could only 


\begin{tabular}{|c|c|c|c|c|c|c|c|c|c|c|c|c|}
\hline \multirow[t]{4}{*}{ Region } & \multirow{2}{*}{\multicolumn{4}{|c|}{ Main effects }} & \multicolumn{8}{|c|}{ Repetition effects } \\
\hline & & & & & \multicolumn{4}{|c|}{ 30-sec delay } & \multicolumn{4}{|c|}{ 3-day delay } \\
\hline & $\mathbf{x}$ & $\mathbf{y}$ & $\mathbf{z}$ & Z-score & $\mathbf{x}$ & y & $z$ & Z-score & $\mathbf{x}$ & y & $z$ & Z-score \\
\hline & \multicolumn{4}{|c|}{ nameable objects > noise } & \multicolumn{8}{|c|}{ repeated < novel } \\
\hline $\begin{array}{l}\text { Occipital } \\
\text { left iO G/FG }\end{array}$ & -36 & -72 & -12 & 4.84 & -26 & -78 & -20 & 2.85 & -26 & -74 & -16 & 2.45 \\
\hline right iO G/FG & 44 & -76 & -12 & 6.15 & 42 & -68 & -16 & 2.07 & 40 & -78 & -12 & 2.98 \\
\hline $\begin{array}{l}\text { Temporal } \\
\text { left FG }\end{array}$ & -44 & -62 & -12 & 5.20 & -44 & -54 & -12 & 2.57 & -40 & -48 & -12 & 2.98 \\
\hline right FG & 36 & -52 & -20 & 3.66 & 44 & -56 & -16 & 3.32 & 46 & -58 & -16 & 2.41 \\
\hline \multirow[t]{2}{*}{$\begin{array}{l}\text { Frontal } \\
\text { left IFG }\end{array}$} & -42 & 28 & 16 & 3.50 & -38 & 28 & 12 & 1.32 (ns) & -40 & 28 & 16 & 2.72 \\
\hline & & & & & \multicolumn{8}{|c|}{ repeated > novel } \\
\hline \multirow[t]{2}{*}{ left insula } & -40 & 8 & 8 & 4.40 & -40 & 4 & 8 & 2.78 & -38 & 6 & 8 & 3.37 \\
\hline & \multicolumn{4}{|c|}{ nonsense objects > noise } & \multicolumn{8}{|c|}{ repeated < novel } \\
\hline $\begin{array}{l}\text { Occipital } \\
\text { left iO G/FG }\end{array}$ & -38 & -80 & -8 & 6.12 & -22 & -84 & 0 & 2.65 & -22 & -74 & -20 & 2.04 \\
\hline right iO G/FG & 40 & -84 & -8 & 6.62 & 34 & -78 & 4 & 2.01 & & & & \\
\hline $\begin{array}{l}\text { Temporal } \\
\text { left FG }\end{array}$ & -38 & -56 & -20 & 3.82 & & & & & & & & \\
\hline right FG & 36 & -52 & -20 & 3.08 & & & & & & & & \\
\hline
\end{tabular}

be explained by the nature of the objects (real or nonsense object) and their presentation history.

\section{RESULTS}

\section{Behavioral data}

The behavioral learning effect was established in a separate study. Stimuli and procedure were the same as described for the fMRI study, except that subjects were instructed to name the real objects aloud in both sessions. The mean naming latency for novel objects was $789 \pm 111.18 \mathrm{~ms}$ (mean \pm s.d.). Priming effects were $137 \mathrm{~ms}$ after 30 seconds $(p<0.001)$ and $54 \mathrm{~ms}$ after 3 days $(p<0.001)$.

\section{fMRI data}

We first identified the areas that showed a significant increase in activity for all nameable objects, and separately identified areas of activity increase for all nonsense objects, relative to a visual noise baseline. As expected, viewing nonsense objects increased activity in ventral occipitotemporal cortex bilaterally. Silently naming

Fig. 1. Group averaged results for nameable objects in occipitotemporal cortex. (a) Posterior regions showing increased activity for silently naming objects as compared to viewing visual noise stimuli (color). W ithin these regions, responses were reduced in bilateral inferior occipital and fusiform gyri for naming repeated objects at both the 30-s and the 3-d delay (pink). Some regions showed reduced responses at the 30-s delay only (purple). (b) G roup averaged event-related hemodynamic responses computed from all voxels within occipitotemporal regions active during object naming showing an effect of object repetition at the 30-s delay (that is, all voxels in pink and purple regions). Shown are regional subject mean responses (symbols) and the regional fitted responses (lines) for novel objects (black), repeated objects at a 30-s delay (light gray), and repeated objects at a 3-d delay (dark gray, dashed line). (c) Same as (b) but for all voxels within occipitotemporal regions active during object naming, showing an effect of object repetition at both the 30-s and the 3-day delay (all voxels in pink regions).
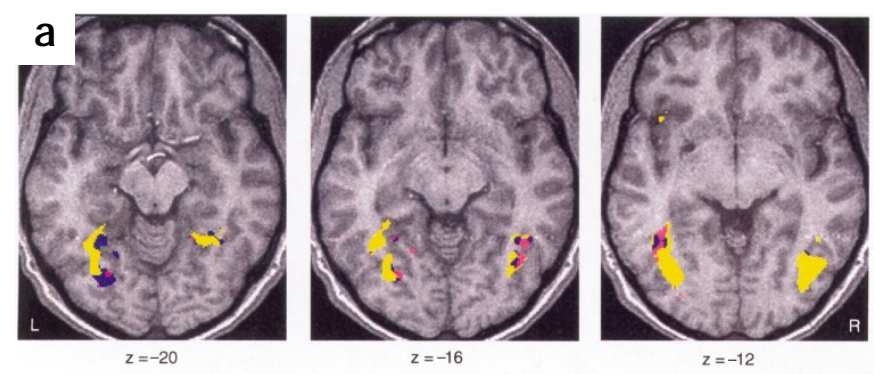

b
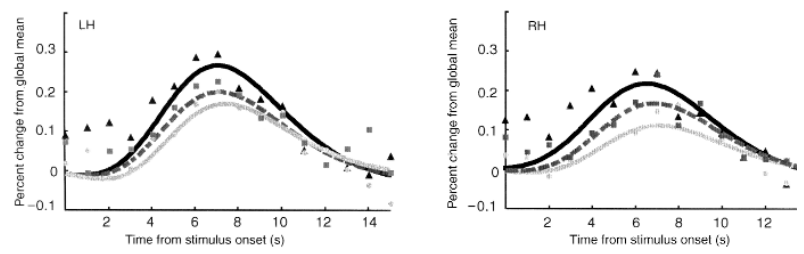

C

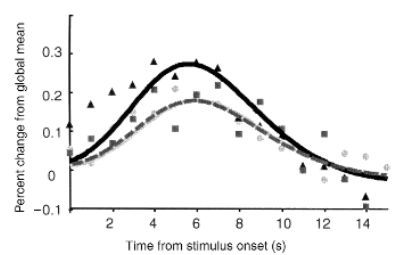

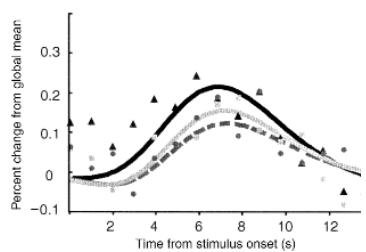


a

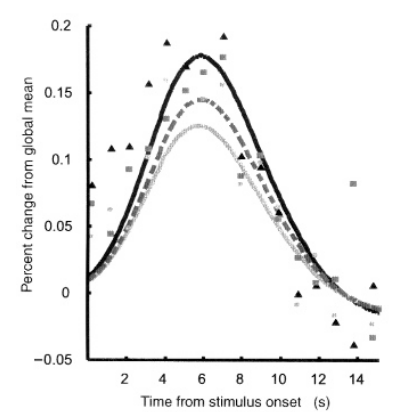

b

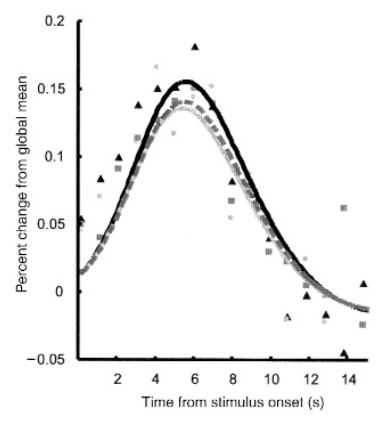

Fig. 2. Event-related hemodynamic responses for nameable objects in occipitotemporal cortex. (a) Group-averaged event-related hemodynamic responses computed from all voxels within occipitotemporal regions responding to silent object naming. Shown are regional subject mean responses (symbols) and the regional fitted responses (lines) for novel objects (black), repeated objects at a 30-s delay (light gray), and repeated objects at a 3-d delay (dark gray, dashed line). (b) Same as (a) but for all voxels within occipitotemporal regions active during object naming showing no significant effect of object repetition at either the 30 second or the 3-d delay (all voxels in yellow regions in Fig. 1a).

real objects increased activity in bilateral ventral occipitotemporal, left inferior frontal, left insula, premotor and cingulate cortices ${ }^{15}$. Within those regions, we tested for significant increases and decreases in activity as a function of object repetition.

In occipitotemporal regions, activity was greatly reduced for nameable objects that were repeated after a 30 -second delay. Within these regions, activity was still significantly reduced after three days (Fig. 1; Table 1). The areas that showed significantly decreased activity were confined to subsets of voxels, centered around the peak activations within the larger region that was active during silent object naming. However, repetition-related decreases seemed to be a general property of the object-responsive cortex (Fig. 2). Decreased neural responses occurred throughout occipitotemporal cortex, but were statistically significant only in those voxels most active during silent object nam-

ing. No voxels were identified that showed significantly increased responses for repeated as compared to novel objects in posterior regions.

To examine whether this repetition priming effect in posterior regions was restricted to objects with pre-existing lexicalsemantic representations, we tested for increases and decreases in activity as a function of nonsense object repetition. For repeated nonsense objects, activity was significantly reduced after 30 seconds in all subjects. This reduction, which was limited to ventral occipital cortex, was also detectable after a three-day delay (Fig. 3). However, although the regions showing decreased activity for nameable objects were in relatively the same location across subjects, locations of the regions showing a significant decrease in activity for nonsense object repetition were more variable across subjects. Nevertheless, the group analysis for nonsense objects showed significant priming effects in small regions of the fusiform gyri of the left and right occipital lobes at the 30-s delay, and the left occipital lobe at the 3-day delay (Table 1). No voxels were identified that showed significantly increased responses for repeated as compared to novel nonsense objects in posterior regions. Thus, a similar pattern of change was observed in posterior cortex for nameable objects and for objects without higher-order representations.

For nameable objects, learning-related changes were also found in left inferior frontal and left anterior insular cortices (Table 1). However, these regions showed opposite patterns of change. Whereas activity in left inferior frontal cortex was reduced (Fig. 4a and b), activity in left anterior insula was increased (Fig. 4c and d). In addition, in contrast with the immediate decrease in activity in occipitotemporal cortex, the changes in anterior regions were mainly observed in response to repetition of objects first seen three days before scanning (Fig. 5). To test the differences in direction and time course of repetition effects in bilateral occipitotemporal, left inferior frontal and left insular cortices, we computed regression coefficients for each subject from regional mean time series after adjustment for

西

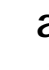

a
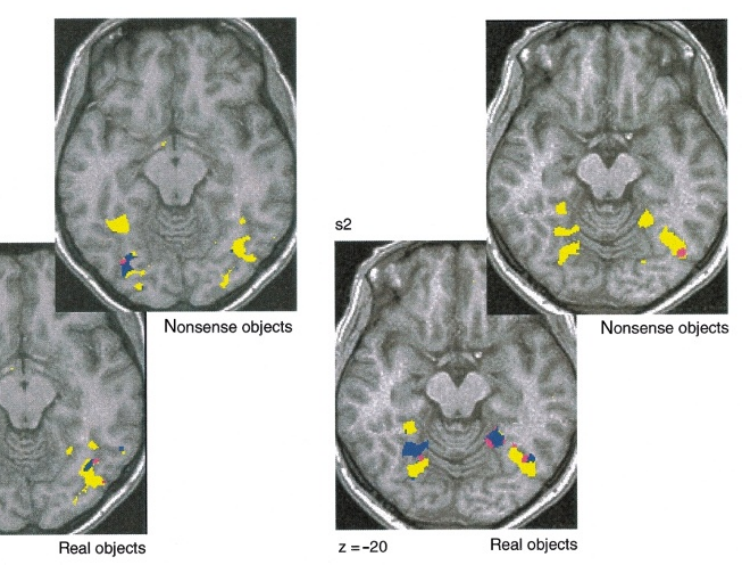

Fig. 3. Individual subject and group averaged results for nonsense objects in occipitotemporal cortex. (a) Examples of posterior regions showing repetition priming for nameable and nonsense objects in two single subjects (s1, s2). In color, regions active when viewing nonsense objects (upper slices), and viewing and silently naming real objects (lower slices) as compared to viewing visual noise stimuli. For both real and nonsense object repetition, responses were reduced at both the 30-s delay and the 3-d delay (pink). Some regions showed reduced responses at the 30 -s delay only (purple). (b) Average event-related hemodynamic responses computed for each of the individual subjects from all voxels active when viewing nonsense objects and showing an effect of nonsense-object repetition at the 30-s delay. Shown are group-averaged regional subject mean responses (symbols), group-averaged regional fitted responses (lines) for novel nonsense objects (black), repeated nonsense objects at a 30 -s delay (light gray), and repeated nonsense objects at a 3-d delay (dark gray, dashed line). (c) Same as (b) but for all voxels active when viewing nonsense objects and showing an effect of nonsenseobject repetition at the 30 -s and the 3-d delay.

\section{b}

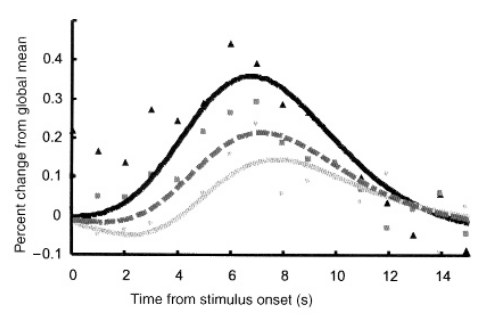

C

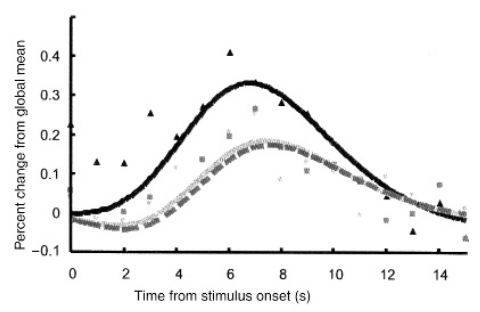


Fig. 4. Group averaged results for nameable objects in anterior regions. Increased activity for silently naming objects as compared to viewing visual noise stimuli (color) was observed in the left inferior frontal and the left insula. (a) In the left inferior frontal gyrus, activity was significantly reduced for naming repeated objects as compared to novel objects at the 3-d delay (green); reduction was not significant at the 30-s delay. (b) Average event-related hemodynamic responses computed from all voxels within the left inferior frontal gyrus active during object naming, showing an effect of object repetition at the 3-d delay (all voxels in green regions). Shown are regional subject mean responses (symbols) and the regional fitted responses (lines) for novel objects (black), repeated objects at a 30-s delay (light gray), and repeated objects at a 3-d delay (dark gray, dashed line). (c) In the left insula, activity was significantly increased for naming repeated as compared to novel objects at both the 30-s delay (orange) and the 3-d delay (orange and red). (d) Same as (b) but for all voxels within the left insula active during object naming, showing an effect of object repetition at the $3-d$ delay.

confounds, and analyzed these data by repeated-measures ANOVA. Voxels were selected for regional computation if they demonstrated both a significant difference from visual noise baseline $(p<0.005)$ and a significant repetition priming effect at one or both delays $(p<0.05)$ in the group analysis. A significant region $\times$ time interaction was obtained for the effects in the left inferior frontal and left insular regions $(p=0.02)$. Whereas activity in left inferior frontal cortex continued to decline as a function of the delay, activity in the left anterior insula increased over time. Pairwise comparisons $(t$-test, $p<0.05)$ showed that both the increase in activity in the left insula and the decrease in the left inferior frontal region were larger at the 3-day delay than at the 30 -second delay. In contrast, the reduction in occipitotemporal activity was largest at the 30 -second delay. That is, whereas occipitotemporal areas showed large decreases in activity after 30 seconds, which were maintained over time, the changes in the left inferior frontal and left insular regions were greater after 3 days than after 30 seconds.

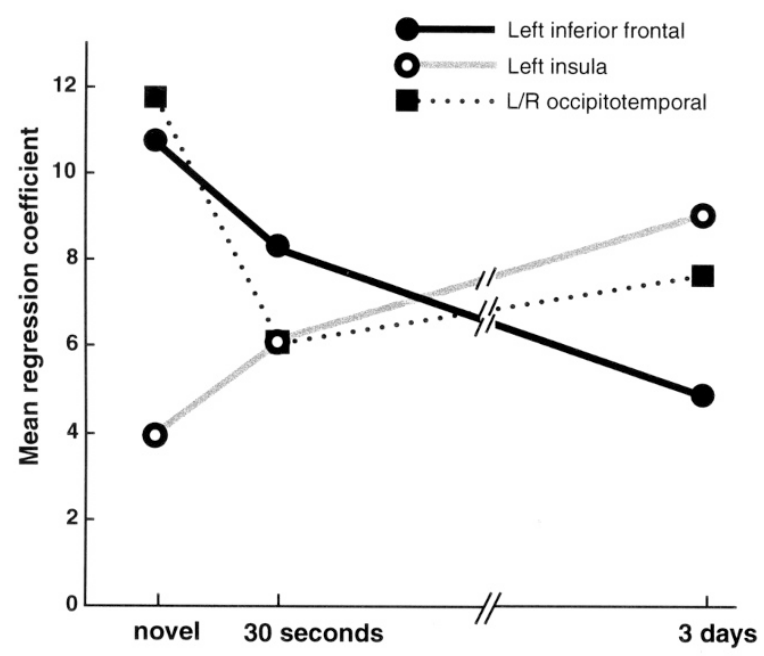

\section{Discussion}

Our findings provided direct evidence for long-lasting cortical plasticity in human adult cortex induced by a single, brief experience with an object. First, visual presentation of a novel object led to a decrease in activity in bilateral occipitotemporal cortex. This decrease occurred quickly (within 30 seconds) and was very long-lasting, even when no previous object representations existed in memory. Second, slower changes occurred in the left inferior frontal and left anterior insular cortices. These changes were opposite in direction: whereas activity in left inferior frontal cortex continued to decline as a function of the delay, activity in the left anterior insula increased over time. The differences in time course and direction of effects strongly suggested that the neural changes in anterior and posterior regions are distinct, and thus may reflect different types of learning.

The occipitotemporal regions that showed repetition priming are part of the ventral, visual processing stream known to be involved in object identification ${ }^{15,17}$. We proposed that long-lasting experience-related facilitation in object identification is mediated by a decreased neural response in the occipitotemporal pathway, possibly reflecting the creation of sparser, object-formspecific representations. Our data were consistent with this hypothesis. Adaptation of neural responses was not restricted to meaningful objects, suggesting that the effect may be located at the level of object form. Moreover, our findings indicate that decreased activity is not simply a short-lasting, general response to stimulus repetition. Rather, this effect is maintained over a rela-

Fig. 5. Estimates of signal amplitude (mean regression coefficients), relative to the visual noise baseline, for each of the object naming conditions: novel, repeated after 30 seconds, and repeated after 3 days. Mean regression coefficients are plotted for regions within the left inferior frontal gyrus (gray line), the left insula (black line) and bilateral occipitotemporal cortex (dotted line); see text for details on statistical procedures. 
tively long period of time, perhaps reflecting a permanent change.

Repetition-related decreases in the amplitude of the fMRI signal could reflect either a decrease in the firing rate of the neurons that responded on initial presentation, or decreased firing of only a subset of these neurons, coupled with maintained responses of a smaller neuronal population. Although our data do not allow us to distinguish between these alternatives, neurophysiological evidence is consistent with the latter view ${ }^{10}$. Our finding that decreased neural responses tended to occur throughout occipitotemporal cortex also suggests that these repetitionsensitive neurons are probably not clustered in distinct areas within the larger object-selective region in occipitotemporal cortex. Clearly, additional studies are needed to directly address these different possibilities.

In addition to the experience-induced decrease in occipitotemporal cortex, distinct changes were observed in left inferior frontal and left insular cortices. The left inferior frontal region, which showed a three-day learning effect, is part of Broca's area, and is involved in semantic and phonological processing ${ }^{18-20}$. Activation of Broca's area in conjunction with the left anterior insula is observed during various speech production tasks ${ }^{21,22}$. Damage to these areas is associated with different types of language impairments, such as aphasia, and apraxia of speech ${ }^{23-25}$. Our data indicated greater recruitment of the left anterior insula in naming repeated as compared to novel objects. At the same time, object naming became less dependent on Broca's area. A similar pattern of practice-related changes in neural activity is observed by brain imaging during a verb generation task $\mathrm{k}^{22}$. That study reported that as the association between a noun and a verb becomes more automatic due to practice, activity in the insula increases, whereas activity in other cortical areas, including Broca's area, is reduced. The present data showed that the effects in Broca's area and left anterior insula were larger at a 3-day delay than at a 30-second delay. Brain imaging studies on skill learning demonstrate that changes in neural activity continue to evolve several hours after a training session has ended ${ }^{26}$. In addition, studies on memory formation demonstrate the necessity of a consolidation period for enduring cortical changes to occur ${ }^{27,28}$. These delayed cortical changes are associated with reorganization of neural representations. We suggest that the time-dependent changes that we observed in the left anterior regions are associated with the strengthening of the link between a novel picture of an object and its lexical representation, and that this process needs time to develop.

There is ample evidence from animal studies that learning involves use-dependent changes in the strength of connections among different groups of neurons ${ }^{29}$. In addition, fMRI data provide evidence for learning-related changes in the functional integration of distinct cortical areas in the human brain ${ }^{30}$. The dynamic changes that we observed in language-related areas, therefore, may reflect reorganization of the neural pathway underlying lexical processing. Our findings suggest that, whereas the retrieval of lexical properties for an object is initially dependent mainly on Broca's area, the role of Broca's area is partly taken over by the left anterior insula when retrieval becomes more automatic with experience. Such changes could be mediated by the direct connections linking Broca's area to insular cortex ${ }^{31}$.

The present findings suggest that the behavioral phenomenon of repetition priming for nameable objects may be mediated by two distinct neural mechanisms. First, an immediate and long-lasting change in posterior regions may reflect the creation of sparser object representations based on a network of neurons tuned to object form. Second, slower developing changes in ante- rior regions may reflect an experience-induced reorganization of the brain circuitry involved in retrieving lexical information about a specific object. Thus, the long-lasting behavioral facilitation of object naming may be mediated by changes at both the perceptual $^{1}$ and lexical levels of representation ${ }^{14}$. Finally, it is important to note that in addition to being able to name objects faster, normal individuals are also capable of explicitly recognizing objects they have previously seen. Thus, it is possible that some of the changes observed in this study may also contribute to explicit recollection. The relationship between these patterns of neural change and implicit and explicit memory processes remains to be determined.

\section{Methods}

Subjects. Eight subjects participated in the fMRI study, and ten subjects participated in the behavioral study. All subjects were right handed and native speakers of English. Subjects gave written informed consent and were compensated for participation. The experimental protocol was approved by the institutional review board of the National Institute of Mental Health.

Stimuli and design. Stimuli included 144 line drawings of real objects, and 144 line drawings of nonsense objects. The sets of novel and repeated nameable objects were matched for word frequency and word length. The assignment of pictures to either the novel or the repeated condition was counterbalanced across subjects. In this way, all pictures contributed equally to each of the conditions. Stimuli were presented in a pseudorandom order, for $200 \mathrm{~ms}$, at a rate of 1 every 2 seconds. In the scanning session, 72 visual-noise stimuli were intermixed with the line drawings to create a low-level, visual baseline condition. Subjects were instructed to look carefully at each picture, and to name the real objects aloud in the session before scanning, and silently in the fMRI-scanning session.

fMRI data collection and analysis. For each subject, 4 time series of 96 whole-brain images were obtained using a gradient-echo, echo-planar scanning sequence (22 axial slices, $5 \mathrm{~mm}$ thick; repetition time, TR, $3 \mathrm{~s}$; echo time, TE, $40 \mathrm{~ms}$; field of view, FOV, $24 \mathrm{~cm}$ ) on a 1.5 Tesla Signa MRI scanner. Images were realigned to correct for interscan movement. A highresolution anatomical scan (three-dimensional spoiled gradient echo sequence; TR, $15 \mathrm{~ms}$; TE, $7 \mathrm{~ms}$, FOV, $24 \mathrm{~cm}$ ) was coregistered to the functional images. Statistical parametric mapping (SPM97, Wellcome Department of Cognitive Neurology, London) was used to analyze fMRI time series. Images were spatially normalized into Talairach space ${ }^{16}$ using a linear transform calculated on the subjects' anatomical images. Images were spatially smoothed with a Gaussian kernel (full-width at half maximum, $6 \mathrm{~mm}$ ), and temporally smoothed with a 4-s Gaussian kernel. Data were ratio normalized to a whole-brain mean signal.

Image time series were analyzed in the context of the general linear model as used in SMP97 (ref. 32). The statistical model included 7 effects of interest (novel real and nonsense objects, repeated real and nonsense objects at a 30-s and a 3-day delay, visual noise) and confounds (a constant for each subject, low-frequency components). The evoked hemodynamic responses for each of the seven event types were modeled as delta functions convolved with a synthetic hemodynamic response function and its temporal derivative ${ }^{32}$. Fixed-effect group analyses and single-subject analyses were done. Parameter estimates, that is, the relative contribution of each event to the mean voxel time series, were obtained with least-squares estimation, and specific effects were tested by applying linear contrasts to the parameter estimates for each event. First, we identified all voxels that showed a significant increase in activity for all nameable objects, and separately for all nonsense objects, relative to a visual noise baseline ( $p<0.005$, uncorrected). Repetition-specific effects were then tested at the voxels that showed a main effect of object presentation, by applying linear contrasts to the parameter estimates of the novel and repeated events. The resulting SPM $(\mathrm{t})$ maps were transformed to $\operatorname{SPM}(Z)$ maps, used for display. Averaged regional hemodynamic responses were computed based on the voxels identified in the group 


\section{articles}

analysis for real objects, and based on the voxels identified in the individual subjects for nonsense objects.

\section{ACKNOWLEDGEMENTS}

We thank Linda Chao for assistance in collecting the MRI data, Jill Weisberg for technical assistance, Karl Friston and Jack van Horn for their advice regarding event-related fMRI data analyses, and Jim Haxby and Leslie Ungerleider for comments on the manuscript.

\section{RECEIVED 1 June; ACCEPTED 10 OCTOBER 2000}

1. Tulving, E. \& Schacter, D. L. Priming and human memory systems. Science 247, 301-306 (1990).

2. Mitchell, D. B. \& Brown, A. S. Persistent repetition priming in picture naming and its dissociation from recognition memory. J. Exp. Psychol. Learn. Mem. Cogn. 14, 213-222 (1988).

3. Cave, B. C. Very long-lasting priming in picture naming. Psychol. Sci. 8, 322-325 (1997).

4. Cave, B. C. \& Squire, L. R. Intact and long-lasting repetition priming in amnesia. J. Exp. Psychol. Learn. Mem. Cogn. 18, 509-520 (1992).

5. Recanzone, G. H. in The New Cognitive Neurosciences (ed. Gazzaniga, M. S.) 237-247 (MIT Press, Cambridge, Massachusetts, 2000).

6. Miller, E. K. \& Desimone, R. Parallel neuronal mechanisms for short-term memory. Science 263, 520-522 (1994).

7. Ringo, J. L. Stimulus specific adaptation in inferior temporal and medial temporal cortex of the monkey. Behav. Brain Res. 40, 191-197 (1996).

8. Squire, L. R. et al. Activation of the hippocampus in normal humans: A functional anatomical study of memory. Proc. Natl. Acad. Sci. USA 89, 1837-1841 (1992).

9. Buckner, R. L. et al. Functional-anatomic correlates of object priming in humans revealed by rapid presentation event-related fMRI. Neuron 20 285-296 (1998).

10. Desimone, R. Neural mechanisms for visual memory and their role in attention. Proc. Natl. Acad. Sci. USA 93, 13494-13499 (1996).

11. Wiggs, C. L. \& Martin, A. Properties and mechanisms of perceptual priming. Curr. Opin. Neurobiol. 8, 227-233 (1998).

12. Levelt, W. J. M. Speaking: From Intention to Articulation (MIT Press, Cambridge, Massachusetts, 1989).

13. Van Turennout, M., Hagoort, P. \& Brown, C. M. Brain activity during speaking: From syntax to phonology in 40 milliseconds. Science 280, 572-574 (1998).
14. Wheeldon, L. R. \& Monsell, S. The locus of repetition priming of spoken word production. Q. J. Exp. Psychol. 44, 723-761 (1992)

15. Martin, A., Wiggs, C. L., Ungerleider, L. G. \& Haxby, J. V. Neural correlates of category-specific knowledge. Nature 379, 649-652 (1996).

16. Talairach, J. \& Tournoux, P. Co-planar Stereotactic Atlas of the Human Brain (Thieme, New York, 1988).

17. Ungerleider, L. G. \& Mishkin, M. in Analysis of Visual Behavior (eds. Ingle, D. J., Goodale, M. A. \& Mansfield, R. J. W.) 549-586 (MIT Press, Cambridge, Massachusetts, 1982).

18. Poldrack, R. A. et al. Functional specialization for semantic and phonological processing in the left inferior prefrontal cortex. Neuroimage 10, 15-35 (1999).

19. Price, C., Indefrey, P. \& van Turennout, M. in Neurocognition of Language Processing (eds. Brown C. M. \& Hagoort, P.) 211-240 (Oxford Univ. Press, Oxford, 1999).

20. Gabrieli, J. D. E., Poldrack, R. A. \& Desmond, J. E. The role of left prefrontal cortex in language and memory. Proc. Natl. Acad. Sci. USA 95, 906-913 (1998).

21. Price, C. J. \& Friston, K. J. Cognitive conjunction: a new approach to brain activation experiments. Neuroimage 5, 261-270 (1997).

22. Raichle, M. E. et al. Practice-related changes in human brain functional anatomy during nonmotor learning. Cereb. Cortex 4, 8-26 (1994).

23. Damasio, H. \& Damasio, A. The anatomical basis of conduction aphasia. Brain 103, 337-350 (1980).

24. Paulesu, E. et al. Is developmental dyslexia a disconnection syndrome? Evidence from PET scanning. Brain 119, 143-157 (1996).

25. Dronkers, N. F. A new brain region for coordinating speech articulation. Nature 384, 159-161 (1996).

26. Karni, A. et al. The acquisition of skilled motor performance: fast and slow experience-driven changes in primary motor cortex. Proc. Natl. Acad. Sci. USA 95, 861-868 (1998).

27. Bontempi, B., Laurent-Demir, C., Destrade, C. \& Jaffard, R. Time-dependent reorganization of brain circuitry underlying long-term memory storage. Nature 400, 671-675 (1999).

28. Zola-Morgan, S. \& Squire, L. R. The primate hippocampal formation: evidence for a time-limited role in memory storage. Science 250, 288-290 (1990).

29. Singer, W. Development and plasticity of cortical processing architectures. Science 270, 758-764 (1995).

30. Büchel, C., Coull, J. T. \& Friston, K. J. The predictive value of changes in effective connectivity for human learning. Science 283, 1538-1541 (1999)

31. Augustine, J. R. Circuitry and functional aspects of the insular lobe in primates including humans. Brain Res. Rev. 22, 229-244 (1996).

32. Friston, K. J. et al. Event-related fMRI: Characterizing differential responses. Neuroimage 7, 30-40 (1998). 